2. A prospective, comparative, randomized study to evaluate the safety and efficacy of scalp block with bupivacaine and clonidine versus bupivacaine only for supratentorial craniotomies

\author{
Shrikanta Oak, Anjana Wajekar, Anita Shetty, Madhu \\ Garasia
}

Department of Anesthesiology, GSMC and KEM Hospital, Parel, Mumbai, Maharashtra, India

Background: Scalp blocks combined with general anesthesia reduce pin and incision response, along with providing stable perioperative hemodynamics and analgesia. Clonidine has proved to be a valuable additive in infiltrative blocks. We studied the efficacy and safety of addition of clonidine $2 \mathrm{mcg} / \mathrm{kg}$ to scalp block with $0.25 \%$ bupivacaine (Group A) versus plain $0.25 \%$ bupivacaine (Group B) for supratentorial craniotomies. Materials and Methods: Sixty patients were randomly divided into Groups A and B. All the patients received propofol based general anesthesia. Intra-operatively, propofol infusion was maintained at 75 to $100 \mathrm{mcg} / \mathrm{kg} / \mathrm{hr}$ up to dura closure and reduced to $25-50 \mathrm{mcg} / \mathrm{kr} / \mathrm{hr}$ up to skin closure with atracurium infusion stopped at dura closure. Heart Rate (HR) and mean arterial pressure (MAP) were monitored at pin insertion, at 5 minute intervals from incison till dura opening, at 15 minute interval till dura closure and again at 5 minute interval up to skin closure, Fentanyl $0.5 \mu \mathrm{g} / \mathrm{kg}$ IV was given if a $20 \%$ increase in either HR and/or MAP was observed. Postoperative hemodynamics and verbal rating scores (VRS) were recorded. When the VRS score increased above 4, rescue analgesia was given. Any complications were noted. Results: Group B showed a significant increase in hemodynamic variables during the perioperative period as compared to group A $(P<0.05)$. Addition of clonidine $2 \mathrm{mcg} / \mathrm{kg}$ in the infiltrative block potentiated the block action with analgesia lasting $960 \pm 120$ minutes. Only patient in group A had bradycardia which responded to glycopyrrolate. Conclusion: Addition of clonidine to scalp block provided better perioperative hemodynamic stability and significantly prolonged analgesia.

3. Acute kidney injury in post operative neurosurgery patients in ICU: A comparison between the RIFLE and the AKIN classifications

Vidit Joshi, R. Ramprasad, Rakhee Goyal

Department of Anesthesiology, AFMC, Pune, Maharashtra, India
Background: Acute kidney injury (AKI) is a known complication after major surgery. Early recognition of AKI can reduce morbidity and mortality. AKI have $>30$ definitions and therefore the wide variation in definitions has made it difficult for comparisons and conclusions. Recently four new definitions of Acute Kidney Injury have been proposed RIFLE, AKIN, KDIGO and WRF of which later two mainly based ON AKIN criteria. Materials and Methods: To compare the RIFLE (Risk, Injury, Failure, Loss and End-stage Renal Failure) and AKIN (Acute Kidney Injury Network) criteria for diagnosis of AKI after Neurosurgery in postoperative ICU patients and associated risk factors in the development of AKI. This is a prospective cohort study of 72 patients from December 2012 to September 2013 with post-op ICU stays $>24$ hours. Other factors contributing to the onset of renal failure were analyzed. Statistical data was analyzed using SPSS 17. Results: The incidence of AKI using the AKIN and RIFLE criteria was 33.33\% and $20.83 \%(P<0.001)$ respectively, but individual patients were classified differently. Diabetes mellitus, Hypoalbuminemia, contrast use, blood loss intra-op, duration of surgery, low pre-op creatinine, number of ventilatory days and APACHE II score were positively associated with AKI. Conclusion: The incidence of AKI in critical patient varies depending on the definition used. The stepwise incremental mortality risk by AKI severity stage is better in AKIN. Use of Diuretics and IV fluids must be considered for accurate identification and staging of AKI. AKIN applied in patient undergoing Neurosurgery without correction of Sr Creatinine for fluid balance may lead to over diagnose AKI.

\section{Efficacy of dexmedetomidine infusion for conscious sedation in patients undergoing awake craniotomy: A preliminary study}

\author{
Charu Mahajan, Girija Prasad Rath¹, Gyaninder Pal \\ Singh $^{1}$, Parmod Kumar Bithal ${ }^{1}$
}

\section{SGPGIMS, Lucknow, India; ${ }^{1}$ AlIMS, New Delhi, India}

Background: The anesthetic goal for awake craniotomy is not only to maintain adequate sedation, analgesia, respiratory and hemodynamic stability but also to provide an awake yet co-operative patient for proper neurological testing. Dexmedetomidine, an $\mathrm{a}_{2}$-agonist, has advantages of being a minimal respiratory depressant with cardiac stabilization properties. Hence, we carried out this study to evaluate the efficacy of dexmedetomidine sedation for awake craniotomy. Materials and Methods: All patients with age above 18 years who underwent awake craniotomy for intracranial tumur surgery under MRI guidance were enrolled. The patients who were un-cooperative, had difficult airway and had medical conditions 